\title{
A Progressive Garbage Collection Scheme Based on Hotness of Valid Pages for NAND Flash Memory
}

\author{
Mingyang LI \\ Tianjin Airport Economic Area \\ Tianjin, China \\ e-mail: 1my9342@163.com
}

\author{
Yonghong ZENG \\ Tianjin Airport Economic Area \\ Tianjin, China \\ e-mail: zyher1974@163.com
}

\begin{abstract}
With the popularity of NAND flash memory, exploiting garbage collection techniques to improve the lifetime of NAND flash memory has become a critical issue in the design of flash translation layer (FTL). However, the existing garbage collection theories always focus on the selection of victim block, but the negative impact on the degree of wear-leveling and the garbage collection overhead is ignored. This paper proposes a progressive garbage collection (PGC) scheme based on the hotness of valid pages. PGC scheme improves the method of choosing the victim block to balance the garbage collection overhead and the degree of wear-leveling and the hotness of valid pages is defined in a new way. Experimental results show that the proposed scheme is better than the existing garbage collection schemes in terms of the number of copy operation, the erase number and the degree of wear-leveling.
\end{abstract}

Keywords-NAND flash memory; garbage collection; wear-leveling; FTL

\section{INTRODUCTION}

Accompany with the rapid development of flash manufacturing technology, the solid state disk (SSD) which uses NAND flash memory as its storage medium has gradually replaced the traditional magnetic disk. NAND flash memory shows many prominent advantages, such as non-volatility, shock resistance, small size and low power consumption [1]. And NAND flash memory slowly becomes the main storage device in the fields of electronic engineering, embedded development and computer technology.

However, NAND flash memory has obvious shortcomings [2]. The traditional magnetic disk deletes and updates the data on its original address while the block is the minimum unit of an erase operation in NAND flash memory. If some data needs to be modified, new data will be wrote to other free pages and the pages containing the original data will be marked as invalid and regarded as garbage pages. As times goes by, the garbage pages will accumulate and a garbage collection scheme should be carried out to obtain the free space when the available space reduces below a threshold. Meanwhile, the erase count of every block in NAND flash memory has a limit [3] and it is not expected that only a small portion of all blocks are erased frequently.

Numerous of garbage collection schemes have been proposed from universities and other research establishments. The existing garbage collection theories always focus on the selection of victim block, but the negative impact on the degree of wear-leveling and the garbage collection overhead is ignored [4]. In this paper, a progressive garbage collection (PGC) scheme based on the hotness of valid pages is proposed. It improves the method of selecting a victim block and balances the garbage collection overhead and the degree of wear-leveling. In order to further lower the wear-leveling degree, the number of other free pages which are wrote during the time between the adjoining update operations is utilized to define the hotness of valid pages in victim blocks. Then the hot pages and cold pages will be migrated to different free blocks. The progressive garbage collection scheme and previously proposed ones have been evaluated by means of several simulations. The results present that the proposed scheme is preferable in terms of the number of copy operation, the number of erase operations and the degree of wear-leveling.

The rest of the paper is organized as follows. Section II 
presents an overview of the flash translation layer (FTL) in NAND flash memory. Section III reviews the related work about garbage collection. Section IV illustrates the proposed garbage collection scheme in detail. Section V shows the experimental results and performance evaluation. Finally, conclusions are gave in last section.

\section{BACKGROUND}

NAND flash memory is a kind of non-volatile storage medium with high write and read performance. Though NAND flash memory has a number of outstanding advantages, the upper file system can not access NAND flash memory directly like the traditional magnetic disk. In order to overcome the drawback, a flash translation layer (FTL) has been employed. FTL maps the logical block addresses of read or write operation from the file system to the physical addresses corresponding to specific pages in NAND flash memory [5], making the upper file system treat NAND flash memory as a magnetic disk.

The distinctive physical characteristics of NAND flash memory bring it some shortcomings [6]. Due to the erase-before-write hardware constraint, NAND flash memory must adopt an out-of-place update scheme to improve the performance of NAND flash memory. As a result, several versions of data of the file system might coexist in different flash pages even if only one version is valid. When there are too many out-of-date flash pages, FTL should trigger the garbage collector to erase some blocks with the aim of obtaining the free space. In addition, every block in NAND flash memory has a fixed erase-count-maximum, typically 10,000 100,000 times. It is necessary for FTL to implement a wear-leveling algorithm so that all the blocks can be erased evenly. In summary, the key components of FTL are address translator, garbage collector and wear-leveler [7].

At present, FTL has been a hot topic in NAND flash memory research. Many scholars focus on the study of garbage collection scheme and wear-leveling algorithm because an excellent garbage collection scheme or wear-leveling algorithm could provide a better performance of NAND flash memory. The existing garbage collection schemes are generally at the expense of the high garbage collection overhead and the waste of internal memory, besides, the degree of wear-leveling is not good enough. In this instance, a progressive garbage collection scheme should be proposed so as to ultimately minimize the performance overhead, the memory waste and reduce the degree of wear-leveling.

\section{RELATED WORK}

In general, garbage collection scheme aims at improving the efficiency of the reclaim operation to obtain more available space and reduce the erase count of all flash blocks. A number of garbage collection schemes have been proposed for NAND flash-based product in the field of electronic engineering and embedded development.

$\mathrm{Wu}$ presented a garbage scheme called greedy (GR) scheme [8]. It selects the block with the maximum invalid pages as the victim block in the hope of obtaining the maximum amount of space at a minimum cost. This scheme is characterized by simple and high reclaim efficiency. However, the GR scheme does not consider the erase count of every block when it seeks the victim block. If there is quite a little valid data which is rarely updated (cold data) in some blocks, these blocks have a slim chance to be selected by GR scheme. Accordingly, NAND flash memory may suffer a high degree of wear-leveling.

In order to solve the problem, a cost-benefit (CB) garbage collection scheme is proposed by Kawaguchi [9]. The CB scheme employs a formula to compute a value of each block and the block with the maximum value will be selected. The formula is as follow:

$$
\operatorname{age} \times(1-\mu) / 2 \mu
$$

where age is the elapsed time since the last page invalidation of this block and $\mu$ represents the ratio of valid flash pages in this block. The term $1-\mu$ represents the ratio of the obtained free space after erasing the block. The term $2 \mu$ is the cost that reading the valid data from the block and writing them to another free block. Because of the term age, cold flash blocks have a higher chance to be selected than hot flash blocks. The CB scheme has a better performance than the 
GR scheme as a result of considering the age of flash blocks, but the degree of wear-leveling is still high.

To further reduce the degree of wear-leveling, Chiang improved the $\mathrm{CB}$ scheme and designed a cost-age-time (CAT) garbage collection scheme [10]. Compared with the CB scheme, it gives consideration to the erase count of each flash block and selects the block with maximum value computed by formula (2).

$$
\frac{\text { age }}{n} \times(1-\mu) / 2 \mu \text {. }
$$

where $\mathrm{n}$ is the erase count of the flash block.

In order to further improve the performance, Han proposed a cost-age-time with age-sort (CATA) garbage collection scheme [11]. It was developed from the CAT scheme and the formula was changed as follow:

$$
\frac{\text { age }}{n} \times(1-\mu) /(1+\mu)
$$

Moreover, the CAT and CATA schemes separate cold valid pages from hot ones and distribute them to different free blocks so as to further reduce the garbage collection overhead.

\section{A Progressive Garbage Collection Scheme BASEd ON Hotness of VALId PAGES}

This section demonstrates the improvement in choosing the victim block firstly. Then a method used to compute the hotness of the valid pages in victim block and distinguish the cold and hot data is shown.

\section{A. The Improvement in Choosing the Victim Block}

According to the previous research, it is obvious that the victim block should be characterized by high percentage of invalid flash pages, low erase count. And the victim block should also have period of time not to update. Reclaiming such victim blocks is beneficial to lower the degree of wear-leveling.

In previous research, the term age is used to describe the life cycle of each flash block and it is helpful to improve the performance of garbage collector. However, if there is few pages marked as invalid since the last page invalidation of a block, recording the time-data will consume much internal memory. This page proposes a more reasonable description. During the time that a block has no page invalidation, the system may invalidate the pages of other blocks. Hence, the number of the pages which are invalidated in other flash blocks since the most recent invalidation, using $\mathrm{NP}_{\mathrm{i}}$ to stand for it, can replace the term age to describe the life cycle of the $\mathrm{i}$-th block. It is more accurate and recording the $\mathrm{NP}_{\mathrm{i}}$ data occupies less internal memory.

Among the three basic operations of NAND flash memory, the cost of write operation is much higher than that of read operation and the cost to erase a block is much higher than to write a block. The difference between the cost of reclaiming two blocks is just because of the migration cost that write the valid data to other free space. And it is less important than the erase cost. Therefore, this paper only gives consideration to the erase cost and defines it as " 1 ". Then the benefit of reclaiming a victim block could be toke as " $1-\mu$ ", where the term $u$ is the ratio of valid flash pages in this block.

Considering the influence of erase count on garbage collection, a new concept called life-loss-index (LLI) of a flash block is introduced in this paper and it is described as follow:

$$
L L I_{i}=1-\log _{E} E_{i}
$$

where $\mathrm{E}$ is the maximum of erase count of a flash block and $E_{i}$ stands for the recent erase count of the i-th block. The value of $\operatorname{LLI}_{\mathrm{i}}$ is between 0 and 1 and the smaller the value, the higher degree of life loss of the i-th block is it.The victim block should has a large life-loss-index.

In order to choose the best victim block, the paper employs a formula to compute values of flash blocks. The formula is shown below:

$$
\text { Value }_{i}=(1-\mu) \times L L I_{i} \times \log _{N P} N P_{i}
$$

where NP represents the fixed number of pages in a block 
and it is used to avoid that the $\mathrm{NP}_{\mathrm{i}}$ is too large to influence the accuracy of the Value ${ }_{i}$. The garbage collector should choose the block with the maximal value as the victim block when there is not enough space in the NAND flash memory.

\section{B. The Method to Distinguish the Cold and Hot Data}

In a victim block, it is possible that the cold valid data is coexist with the hot valid. In order to further reduce the garbage collection overhead and the degree of wear-leveling, the cold valid data and the hot valid data should be distributed to different target blocks. And then it is significant to find a method to define the hotness of valid pages.

If a valid page contains hot data, the times of writing to other free pages between its two update operations should be less than a cold valid page. Therefore, the hotness of each valid page is estimated as follow:

$$
\begin{gathered}
H_{i}=\alpha \times \log _{N P} P_{i}+(1-\alpha) \times H_{i-1} . \\
H_{1}=\log _{N P} P_{1} .
\end{gathered}
$$

where $P_{i}$ is the number of other free pages which are wrote during the time from (i-1)-th to i-th update operations of the valid page. The term $\mathrm{H}_{\mathrm{i}}$ represents the hotness of the valid page after the data in this page has been updated i-times. The coefficient $\alpha$ is a weighting factor and its value is between 0 to 1 . The smaller the $\mathrm{H}_{\mathrm{i}}$, the more hot the valid page is it.

Next, the degree of wear-leveling is defined.

$$
D=E_{\max }-E_{\min }
$$

where $E_{\max }$ is the maximum erase count and $E_{\min }$ is the minimum erase count. When there is not enough space in the NAND flash memory, the garbage collector will be triggered and firstly it checks that whether the degree of wear-leveling is larger than a predefined threshold $\mathrm{D}_{\mathrm{th}}$. If so, the block with the least erase count will be chose as the victim block. Otherwise, the garbage collector will choose a victim block according to the Value $\mathrm{i}_{\mathrm{i}}$. This design can make sure that the degree of wear-leveling is controlled within a certain range at all time.

In order to further reduce the garbage collection overhead and lower the degree of wear-leveling, the hot valid pages and the cold ones in victim block should be migrated to different free blocks. After a victim block is chosen, the garbage collector first checks whether a valid page is cold or hot. If the hotness of a valid page is larger than the average hotness of all valid pages in victim block, it is regarded as a cold valid page. Otherwise, it is considered as a hot valid page. Then the hot valid pages will be distributed to a free block with the smallest erase count in free-block linked list and the cold valid pages will be migrated to a free block with the largest erase count. The new free block produced by garbage collection scheme will be inserted into the linked list by the method of bisection on the basis of its erase count.

\section{PERFormance EVAluation}

In this section, the capability of the proposed garbage collection scheme is evaluated.

To assess the performance of the PGC scheme, a 64M NAND flash memory is simulated and the specifications is shown in Table 1. In the experiments, GR scheme, CB scheme, CAT scheme, CATA scheme are used to compared with the proposed PGC scheme in this paper. And the performance metrics are the number of copy operations, the number of erase operations and the degree of wear-leveling.

TABLE I. PARAMETERS OF THE EXPERIMENT

\begin{tabular}{|c|c|c|c|c|}
\hline Page size & Block size & Read time & Write time & Erase time \\
\hline $2[\mathrm{~KB}]$ & $128[\mathrm{~KB}]$ & $25[\mu \mathrm{s}]$ & $200[\mu \mathrm{s}]$ & $2000[\mu \mathrm{s}]$ \\
\hline
\end{tabular}




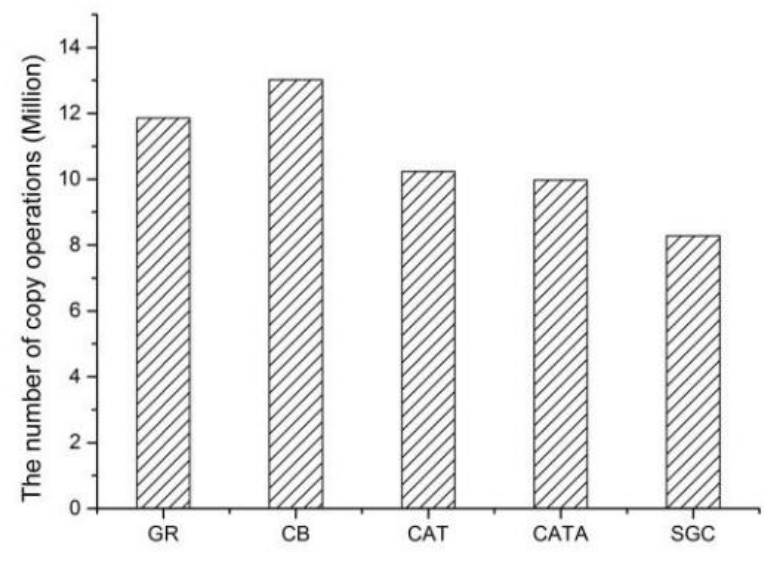

Figure 1. The number of copy operations

Figure 1 shows the simulation results of different garbage collection schemes on the part of the number of copy operations. The number of copy operations relies on the number of the valid pages in the victim blocks. Due to that GR scheme always selects the block with the least valid pages as a victim block, it brings less copy operations than CB scheme. Compared with GR scheme, CAT scheme and CATA scheme generate less copy operations, but the reductions are not significant. The proposed PGC scheme obtains the best performance among the five garbage collection schemes, reducing the number of copy operations by about thirty percent. This is simple because the cold valid pages and the hot ones are distributed to different free blocks.

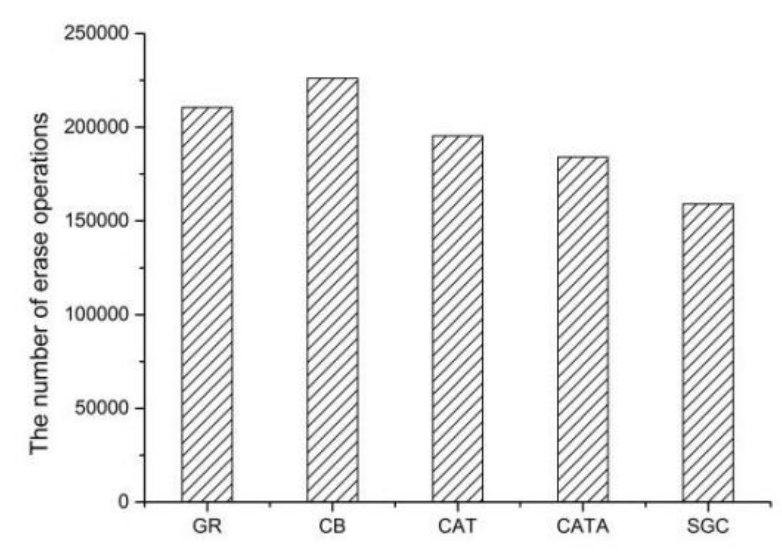

Figure 2. The number of erase operations

Figure 2 shows the simulation results of different garbage collection schemes on the part of the number of erase operations. The erase operations are incurred by triggering the garbage collector when the free space is not enough. Every time the garbage collector is triggered, the number of erase operations increases. The proposed PGC scheme computes the hotness of every valid pages in victim block. Then the hot valid pages are separated from the cold valid pages and PGC scheme migrates the hot data to a young free block. This design is helpful for the subsequent garbage collection operations to obtain more free space than other garbage collection schemes. Hence, the proposed PGC scheme triggers the garbage collector fewer times and has less erase operations.

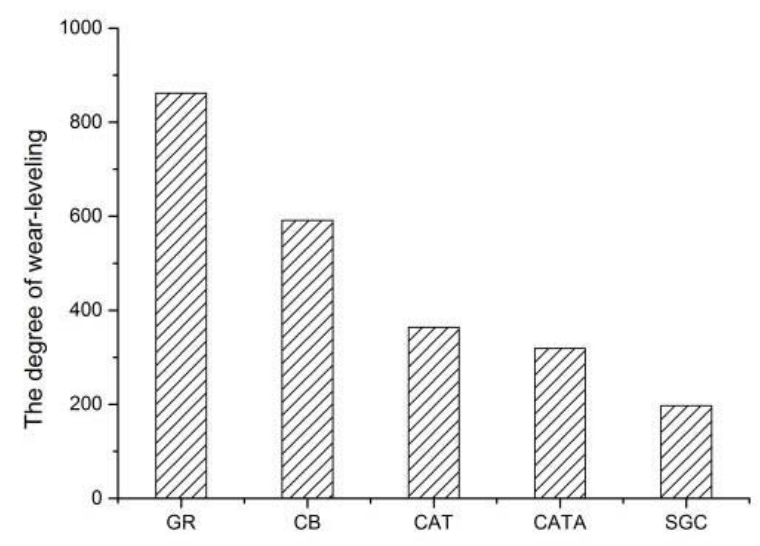

Figure 3. The degree of wear-leveling

Figure 3 shows the simulation results of different garbage collection schemes on the part of the degree of wear-leveling. It can be seen that GR scheme has the highest degree of wear-leveling as a result of singly considering the ratio of valid flash pages of each block and $\mathrm{CB}$ scheme still has a high degree of wear-leveling though it takes the age of flash blocks into account. In contrast, CAT scheme, CATA scheme and the proposed PGC scheme have better performance in this respect and the PGC scheme performs best. This is due to that the PGC scheme not only considers comprehensive factors to choose a victim block, but also checks the degree of wear-leveling when it triggers the garbage collector. Moreover, the migration strategy based on the hotness of the valid pages lowers the degree of wear-leveling effectively. Therefore, as shown in Fig. 3, the proposed PGC scheme 
obtains the best performance in terms of the degree of wear-leveling compared with other garbage collection schemes.

\section{CONCLUSION}

This paper proposes a progressive garbage collection scheme based on the hotness of valid pages. In order to get a balance between the garbage collection overhead and the degree of wear-leveling, the paper improves the method of choosing a victim block. Meanwhile, the proposed scheme finds a way to define the hotness of every valid page in victim block and then migrates the cold valid pages and hot ones into different target free blocks to further reduce the garbage collection overhead and the degree of wear-leveling. The capability of the proposed design is verified by a series of experiment and the results are encouraging. In the future work, the proposed PGC scheme will be optimized in terms of computational cost and computing time.

\section{REFERENCES}

[1] Jeong J, Song Y H. A technique to improve garbage collection performance for NAND flash-based storage systems [J]. IEEE
Transactions on Consumer Electronics, 2012, 58(2):470-478.

[2] Murugan M, Du D H C. Rejuvenator: A static wear leveling algorithm for NAND flash memory with minimized overhead [C]// Mass Storage Systems \& Technologies. IEEE, 2011:1-12.

[3] Ahn S, Hyun S, Kim T, et al. A compressed file system manager for flash memory based consumer electronics devices [J]. IEEE Transactions on Consumer Electronics, 2013, 59(3):544-549.

[4] Lin M, Yao Z. Dynamic garbage collection scheme based on past update times for NAND flash-based consumer electronics [J]. IEEE Transactions on Consumer Electronics, 2015, 61(4):478-483.

[5] Hu Y, Dong X. A kind of FTL Scheme Which Keeps the High Performance and Lowers the Capacity of RAM Occupied by Mapping Table [C]// IEEE International Conference on Networking, Architecture and Storage. IEEE Computer Society, 2016:1-2.

[6] Kwon S J, Ranjitkar A, Ko Y B, et al. FTL algorithms for NAND-type flash memories [J]. Design Automation for Embedded Systems, 2011, 15(3):191-224.

[7] Chen F H, Yang M C, Chang Y H, et al. PWL: A progressive wear leveling to minimize data migration overheads for NAND flash devices [C]// Design, Automation and Test in Europe. 2015:1209-1212.

[8] Wu M, Zwaenepoel W. eNVy: a non-volatile, main memory storage system [C]// Workstation Operating Systems, 1993. Proceedings. Fourth Workshop on. IEEE, 1993:116-118.

[9] Kawaguchi A, Nishioka S, Motoda H. A flash-memory based file system [C]// Usenix 1995 Technical Conference Proceedings. USENIX Association, 1995:13-13.

[10] Chiang M L, Chang R C. Cleaning policies in mobile computers using flash memory [J]. Journal of Systems \& Software, 1999, 48(3):213-231

[11] Han L Z, Ryu Y, Yim K S. CATA: A Garbage Collection Scheme for Flash Memory File Systems [C]// Ubiquitous Intelligence and Computing, Third International Conference, UIC 2006, Wuhan, China, September 3-6, 2006, Proceedings. DBLP, 2006:103-112. 